\title{
Building a fault tolerant application using the GASPI communication layer
}

\author{
Faisal Shahzad*, Moritz Kreutzer*, Thomas Zeiser*, Rui Machado ${ }^{\dagger}$, \\ Andreas Pieper ${ }^{\ddagger}$, Georg Hager* and Gerhard Wellein* \\ *Erlangen Regional Computing Center, University of Erlangen-Nuremberg Erlangen, Germany \\ ${ }^{\dagger}$ Fraunhofer Institute for Industrial Mathematics ITWM, Fraunhofer Platz 1, Kaiserslautern, Germany \\ \#nstitute of Physics, University of Greifswald, Greifswald, Germany
}

\begin{abstract}
It is commonly agreed that highly parallel software on Exascale computers will suffer from many more runtime failures due to the decreasing trend in the mean time to failures (MTTF). Therefore, it is not surprising that a lot of research is going on in the area of fault tolerance and fault mitigation. Applications should survive a failure and/or be able to recover with minimal cost. MPI is not yet very mature in handling failures, the User-Level Failure Mitigation(ULFM) proposal being currently the most promising approach is still in its prototype phase.

In our work we use GASPI, which is a relatively new communication library based on the PGAS model. It provides the missing features to allow the design of fault-tolerant applications. Instead of introducing algorithm-based fault tolerance in its true sense, we demonstrate how we can build on (existing) clever checkpointing and extend applications to allow integrate a low cost fault detection mechanism and, if necessary, recover the application on the fly. The aspects of process management, the restoration of groups and the recovery mechanism is presented in detail. We use a sparse matrix vector multiplication based application to perform the analysis of the overhead introduced by such modifications. Our fault detection mechanism causes no overhead in failure-free cases, whereas in case of failure(s), the failure detection and recovery cost is of reasonably acceptable order and shows good scalability.
\end{abstract}

Keywords: GASPI; GPI; fault detection; fault recovery/tolerance; checkpoint-restart; pre-allocated spare processes

\section{INTRODUCTION}

The advances in computational capacity of HPC clusters have enabled many fields in research and industry to progress far beyond imagination. Still the demand of more computational capacity is never ending. In the recent past, the consistent exponential growth is achieved with the help of extreme levels of hardware parallelism. This causes a severe reduction in mean time to failure (MTTF) of the overall systems and is visible with every new generation of large clusters. For example, the 'Intrepid' BlueGene/P system (debuted as \# 4 on the top500 list of June 2008 [1], installed at the Aragonne National Laboratory) is reported to have the MTTF of 7.5 days [2]. In contrast, a more recent BlueGene/Q cluster 'Sequoia' (debuted as \# 3 according to Nov. 2013 list, installed at Lawrence Livermore National Laboratory) has a node failure rate of 1.25 per day [3]. On the way to exascale machines, the MTTF is expected to reduce to the order of hours or minutes [4], [5]. This indicates an alarming behavior which, if not taken care of, will question the usability of clusters at exascale.
In HPC systems, programs can face many kinds of failures during runtime, e.g., hardware and software faults, silent errors, Byzantine failures, etc. According to [6], 60\% of all failures are attributed to either memory or CPU failures. Such failures, in addition to others, eventually lead to process or node-level failures, which are the focus of this work. The Majority of the literature regarding fault tolerance towards fail-stop failures falls into either one or a combination of the following four categories: algorithm based fault tolerance (ABFT), checkpoint/restart(C/R), message logging, and redundancy [7].

So far most algorithms (and underlying communication models) are built under a comprehensible assumption that the communication partners of every process stay alive and functional during the entire run of the program. Consequently, the failure of even a single process leads to the failure of the whole application. For large scale applications, it is beneficial to drop this assumption. This would mean that the program should continue to run even after the failure of a certain amount of processes, i.e., node failures. This opens a new dimension of research in the field of fault tolerance. In this context, the first step is to build/utilize a communication library that can provide the necessary supporting functionalities for this purpose, i.e., health information of processes, failure detection mechanisms, propagation of failure information to all relevant processes, etc. During the development of MPI 3.0, efforts were underway to introduce process-level failure tolerance [8], but were not eventually successful. The fault tolerant working group in the MPI forum is currently working on the User-Level Failure Mitigation (ULFM) standard proposal and its prototype implementation (based on OpenMPI) for its potential inclusion in the MPI 3.1 standard [9]. Despite the apparent attractiveness of this new approach, it comes with a new set of challenges, i.e., correct and consistent failure acknowledgment, rebuilding communication infrastructure, recovering lost data from failed process(es), etc. These building blocks require extra effort and caution during application development stages.

In this work, we use the GPI-2 [10] implementation of the GASPI specification [11] to build a fault tolerant application that is capable of recovering dynamically from process failures.

The main contributions of our work are as follows:

1) Design and implementation of the fault detection (FD) mechanism to detect fail-stop failures in parallel appli- 
cations. The recovery mechanism is then implemented, which includes the repairing of broken communication infrastructure and the recovery of the lost data of the failed processes.

2) The FD mechanism is applied to a sparse matrix vector multiplication (spMVM) based application, namely Lanczos eigensolver, to showcase its real usage. The necessary algorithmic restructuring is presented in detail. The changes in the underlying spMVM library are also highlighted.

3) For recovering the data of the failed processes, the application-level $\mathrm{C} / \mathrm{R}$ approach is used. Despite the criticism it faces, the recent neighbor node-level $C / R$ optimizations [12], [13] have enabled it to be a good candidate on future exascale systems [4]. We have implemented a neighbor node-level C/R library for GPI2 applications. In case of a node failure, the neighboring nodes are bound to change, thus the C/R library is also made fault aware.

4) A benchmark study has been performed to analyse the overheads involved in this fault tolerance approach. This includes a range of application runtime cases, i.e., overhead in failure-free case and overhead with 1,2,3 failure recoveries, etc. Moreover, the scalibility of the fault detection method is tested with up to 256 nodes.

With this practice, we have developed a fault tolerant application which can heal itself dynamically after the loss of one or more processes. Thus the time for restarting the job manually and wait-time spent in the queueing system for a new job request are avoided. The concept can be applied to other applications with different communication libraries as well when they support fault tolerance. We think that this is a good starting point to gain experience and become aware of the challenges involved and their potential solutions for utilization of such fault tolerant communication libraries.

The paper follows the following structure. Some preliminary definitions and concepts are described in Sect. [II In Sect. III] the GASPI communication interface is briefly introduced along with its fault tolerance features. The design and implementation of our fault tolerance method along with the fault detection mechanism are described in Sect. IV Section $\mathrm{V}$ introduces the benchmark environment and provides application specific details for benchmarks. The overhead analysis of our implementation is presented with benchmarks in Sect. VI A brief summary of the related work is described in Sect. VII. We conclude the paper in Sect. VIII with a short discussion about the challenges of such a fault tolerance approach and possible improvements.

\section{Preliminaries}

There are three central components in application-driven fault tolerance. In this paper, we use the term 'applicationdriven' fault tolerance for an approach where an application can heal itself dynamically despite one or more process failures. The first and foremost component is to have a consistent, accurate and reliable failure detection mechanism. Design and implementation of a fault detector for asynchronous systems is a complex task. A distributed system is termed as 'asynchronous' if there is no upper bound on message transmission delays and process execution time, and all real HPC systems fall into this category. There are two basic properties of a fault detector [14]: 1) Completeness: The crashed processes are suspected by some healthy processes, after a certain amount of time. A strong completeness implies that every failed process is eventually detected by every healthy process. 2) Accuracy: Every detected failure corresponds to a crashed process (no false-positives). The complete satisfaction of both these properties is theoretically impossible [14]. Thus most FD implementations are willing to tolerate some level of inaccuracy but require strong completeness.

The implementation of ULFM relies on detection of faults based on the communication failure between processes. After a failed process is detected, the user can revoke the rest of the communication in a communicator and propagate the failure information about failed processes using ULFM functions. In this work, we choose a different approach where an explicit fault detector monitors the health of every other process. Due to the PGAS nature of communication in GASPI, this brings certain advantages in terms of developing consensus between processes after failure(s) and incurs no overhead in the failurefree case (discussed in detail in Sect. IV].

After a correct failure detection, the second step involves the selection of a communication reconstruction and domain redistribution strategy. There can be two approaches in this context [15]. 1) Shrinking recovery: In this method, the application proceeds with the rest of available processes after failure(s) and requires redistribution of domain. 2) Non-shrinking recovery: This method involves using new process(es) to replace the failed one(s), where the work distribution of the application is not changed after failures. Depending on the application and the communication library, either of these techniques can be beneficial. In our work, we have used a non-shrinking recovery method.

The third stage involves the data recovery of the lost process(es) and continuing further computation. Data recovery can either be done by reading a checkpoint or by using an algorithm based fault tolerance (ABFT) approach. An ABFT approach is by nature highly algorithm-specific and cannot be generalized; therefore, we have opted for application specific node-level C/R approach in our application.

\section{INTRODUCTION TO GASPI AND GPI-2}

GASPI is a communication library for $\mathrm{C} / \mathrm{C}++$ and Fortran. It is based on a PGAS style communication model where each process owns a partition of a globally accessible memory space. GPI-2, the GASPI implementation, takes full advantage of the hardware capability to perform remote direct memory access (RDMA). More importantly, there is a focus on providing truly asynchronous communication to overlap computation and communication, and on thread-safe communication which allows multi-threaded applications with a fine-grained communication and asynchronous execution capability.

GASPI defines a very compact API consisting of onesided communication routines, passive communication, global 
atomics and collective operations. It also defines groups which are similar to MPI communicators and are used in collective operations. Furthermore, there is the concept of segments. Segments are contiguous blocks of memory and can be made accessible (to read and write) to all threads on all ranks of a GASPI program. Data to be communicated is thus placed in such segments.

Given the aforementioned increasing need for fault tolerant applications, GASPI was designed with that in mind. It supports application-driven fault tolerance on the process level. This means that the failure of a single process does not cause the entire program to fail. The program can in fact react to the failure and try to recover by "healing itself". This is achieved with two simple concepts: timeouts and an error state vector.

GASPI provides a timeout mechanism to all potentially blocking procedures. The user provides a timeout (in milliseconds) and the procedure returns after that time if it could not successfully complete. Furthermore, since a timeout does not necessarily imply an error or failure from the remote part, GASPI provides an error state vector that holds the state of processes. The state vector is set after every erroneous, nonlocal operation and can be used to detect failures on remote processes. Currently, each rank can either have a state of GASPI_STATE_HEALTHY or GASPI_STATE_CORRUPT. This error state vector can be queried by the application using gaspi_state_vec_get to determine the state of a remote partner in case of timeout or error.

Along with the previous mechanisms, for the context of our work, we have extended GPI-2 to provide an extra mechanism (not included in the GASPI specification) for faulttolerant applications. The procedure gaspi_proc_ping tests the availability of a particular GPI-2 rank. As the name indicates, a ping message is sent to a particular process. In case a problem is detected, a GASPI_ERROR is returned to which the application can react.

In the next sections, we describe our technique in more detail.

\section{DESIGN AND IMPLEMENTATION}

This section explains the details of our fault detector(FD), its implementation in the algorithm, and the data recovery technique for our implementation. The basic idea behind our implementation is to designate some processes as idle processes' at the start of the computation to facilitate nonshrinking recovery. The remaining processes form the 'worker group' and do computation. The idle processes serves two purposes. One of the pre-determined idle processes serves as a failure detector process. The rest of the idle processes stay idle until FD detects a failure and asks idle process(es) to act as rescue process(es) and join the worker group.

\section{A. Failure Detection and Acknowledgment}

Our fault detection method relies on a dedicated FD process periodically checking the health (i.e., aliveness) of all other processes. The FD process pings every other process via gaspi_proc_ping() and checks for its return value as shown in Listing 1 A successful completion (i.e., return value of

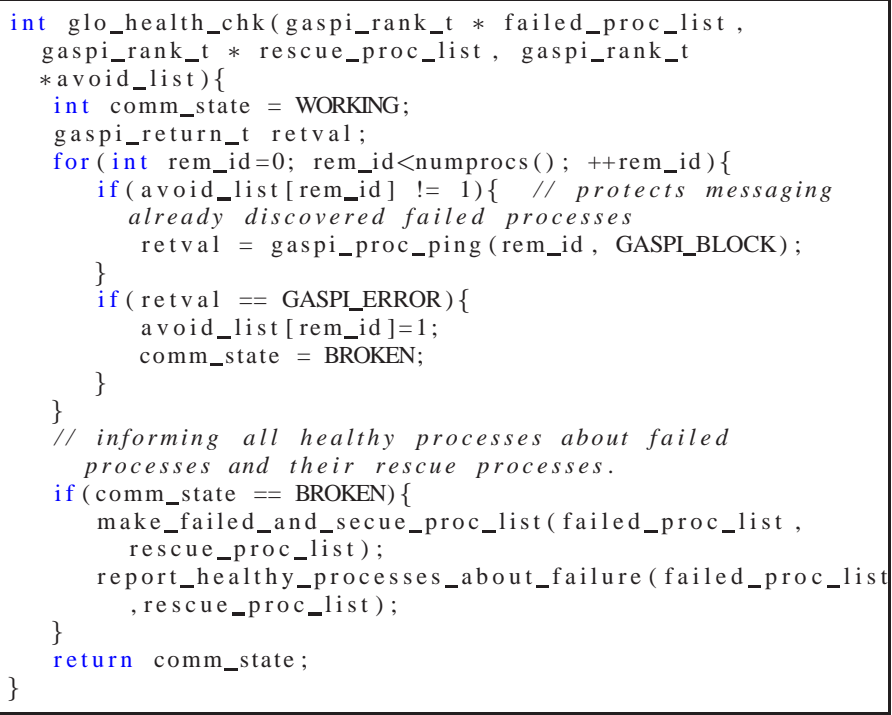

Listing 1: The global health check routine. The fault detector process periodically checks the health of all healthy processes.

GASPI_SUCCESS) of the ping operation implies a healthy process. Figure 1a shows the schematic diagram of such onesided ping based fault-detection. A rational ping frequency can be set by the user depending on the number of processes. After completion of a single cycle around all healthy processes, the FD has an updated global health view. We rely on the fact that each fail-stop failure eventually results in breakage of the communication channel between the FD and the failed process. This consequently leads to the return of ping operation with GASPI_ERROR (shown in Fig. 1b). After detection of failed process(es), the FD process informs all healthy processes about the failed processes as well as their corresponding rescue processes. This is done via one-sided write in the global memory of all healthy processes. Meanwhile, the worker processes communicating directly with the failed processes keep on returning with GASPI_TIMEOUT unless a failure acknowledgment is received. The remaining healthy working processes continue with their work until they also receive a failure acknowledgment signal from the FD process. After failure acknowledgments, no further regular application communication is performed and processes enter the recovery stage of the algorithm. A threaded implementation of the FD process enables it to monitor the health state of multiple processes simultaneously. For exascale systems, multiple FDs can be used to distribute the health check scan of all processes.

a) Fault Detector Properties: The FD process gets an updated global view of all processes' health at the end of each ping cycle around all healthy processes. Thus, a failed process is bound to be detected by the FD process in a finite amount of time. The information about failed processes is then given to all remaining healthy processes. Thus the FD process satisfies the property of completeness. As a rare case, there can be instances that (due to a network related problem) a healthy process is not reachable by the FD process but is accessible by some or all other processes. This will result in a false-positive signal about a processes failure. Thus the property of accuracy 


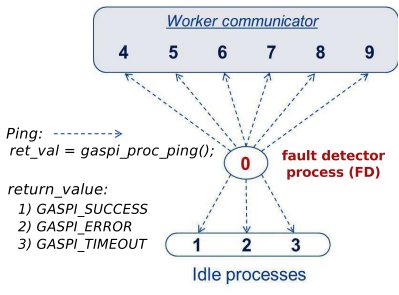

(a) Ping based fault detection by FD process

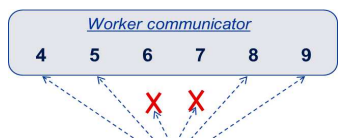

GASPI_ERROR

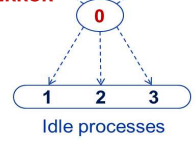

(b) FD detects failure of 6,7 processes and designates 1,2 as rescue processes

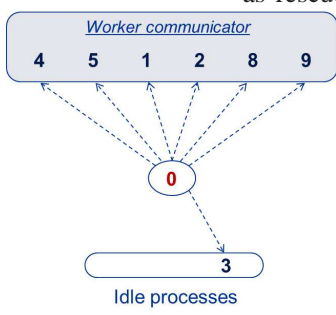

(c) The rescue processes $(1,2)$ join the worker group during failure recovery stage

Figure 1: The working of the fault detector process. The return value of ping is GASPI_SUCCESS for all healthy processes (a), whereas it returns with GASPI_ERROR for failed processes (b). After failure detection, FD informs all processes about failed processes as well as their replacement processes. A new worker group is then built with the help of rescue processes (c).

is only loosely satisfied. A false-positive signal will lead the application to undergo the failure recovery phase but it does not affect the correctness of the program results.

b) Alternate investigated failure detection methods: We also investigated the following failure detection mechanisms:

1) Ping-based all-to-all: In this method each process performs periodic all-to-all pings to detect the failures.

2) Ping-based neighbor level: Each process 'i' periodically pings only its neighboring process ' $i+1$ '. In case a failure is detected at the neighbor level, a ping based all-to-all operation is triggered on all processes to get a global health view.

In both of these approaches, a potential deadlock can arise in the cases where multiple processes detect different sets of failed processes. Reaching a consensus about the identified failures adds further complexity in the algorithm. Moreover an all-to-all ping based approach is not a scalable failure detection method and in failure free cases a certain amount of overhead in both cases is introduced. In contrast, a dedicated FD process with one-sided ping eliminates the potential deadlock situation and causes negligible overhead in failure-free cases.

\section{B. Communication reconstruction}

The FD process propagates the list of failed processes and the list of respective rescue processes to all worker and rescue processes. The worker group is recreated in such a way that the failed processes are left out and the rescue processes are included. Figure $1 \mathrm{~b}$ and $1 \mathrm{c}$ show the schematic of failure

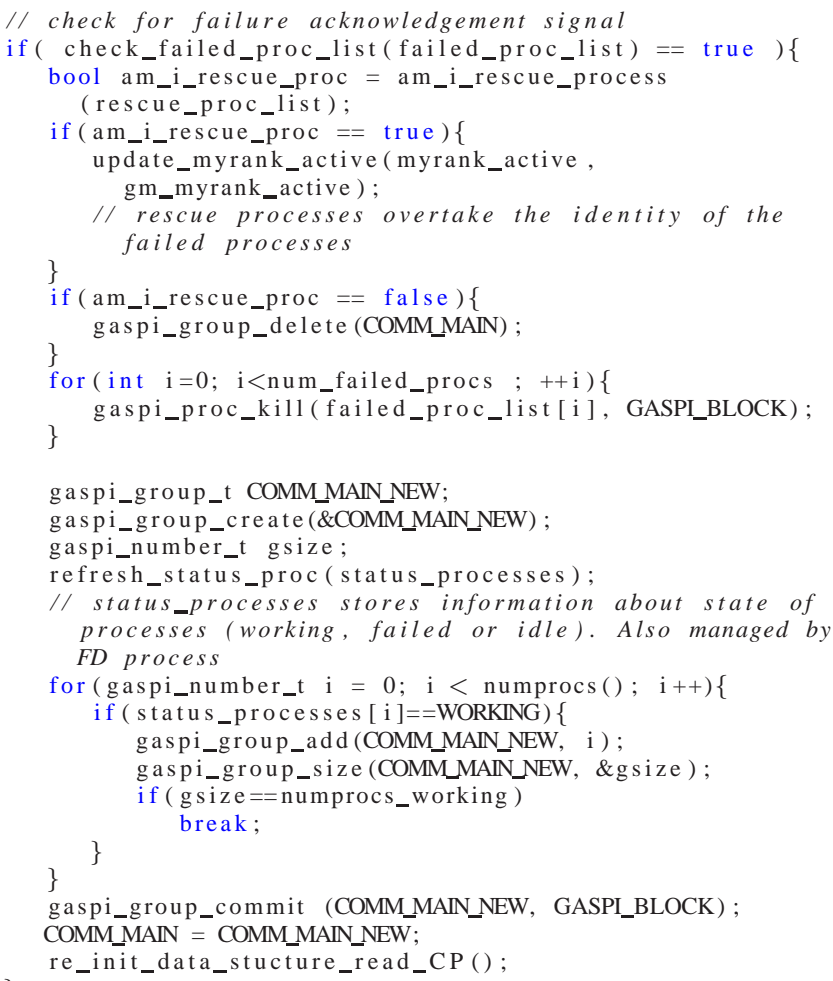

Listing 2: The reconstruction of the working group is performed in case a failure is detected in the health check routine.

acknowledgment and reconstruction of a working group after failure. The steps involved in this recovery mechanism to form a working group are shown in Listing 2. The cases of transient failures and a false-positive detected failures are handled using gaspi_proc_kill in the communication reconstruction phase. It explicitly enforces the processes to die even if they were alive, preventing them from further participation in the application.

\section{Data Recovery: Neighbor node-level checkpoint/restart}

We have utilized the classic application specific C/R technique for the purpose of data recovery in our application. Typically checkpoints are written to the parallel file system (PFS). Writing and retrieving them from PFS is expensive. Therefore we have implemented a library for GPI-2 to take checkpoints at neighbor node-level. Figure 2 shows the schematic of an application utilizing the library. At the init call from the application, the library creates a thread which waits for a signal from the application. At a checkpoint iteration, the application first creates a checkpoint on its local node and signals the library thread after completion. The library thread then copies the local copy of the checkpoint to the neighboring nodes. For a higher degree of reliability, the user can also opt to make infrequent PFS-level copies of the checkpoints. Due to the fault-tolerant nature of the algorithm, the neighboring node of processes can change after failure recovery. This requires the neighbor node-level $\mathrm{C} / \mathrm{R}$ library to be fault aware as well. After the reconstruction of the worker group, the $\mathrm{C} / \mathrm{R}$ library 


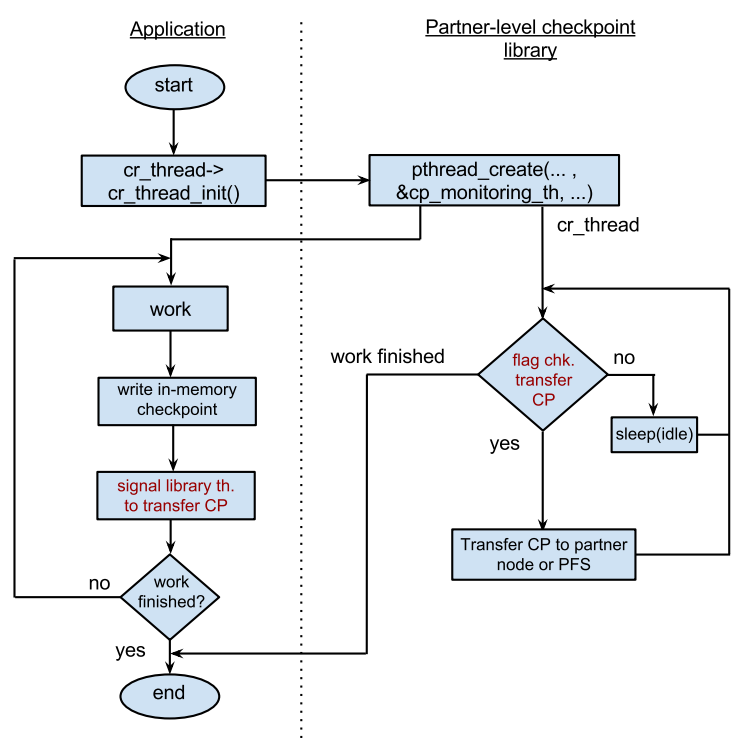

Figure 2: Schematic of the interaction between application and the neighbor node-level checkpointing library.

refreshes its list of neighboring processes based on the failed processes list provided by the application thread.

\section{Application flow}

A flowchart of the modified algorithm is shown in Fig. 3 At the start of the application, the processes are categorized into working and idle processes. One of the idle processes acts as an FD process, whereas the rest stay idle. The worker processes form a worker group and perform computation. The communication routines are checked for a failure acknowledgment signal from the FD process. The FD process periodically checks the global state of the processes. Upon detection of failure(s), it informs all remaining processes which then enter the recovery stage of the algorithm to reconstruct the worker group and restart from a consistent checkpoint. The FD process itself joins the worker group if no idle process is further available.

The current scheme has following restrictions which we plan to address in the future work.

1) The number of failures a program can sustain is equal to the number of idle processes specified at the start of the program.

2) The fault tolerance capability of a program ends if the FD process becomes a worker or encounters a failure itself.

3) Only those network failures can be detected that can be uniformly seen by the effected processes as well as by the FD process.

\section{E. Fault Tolerance Overhead}

Each of the fault tolerance techniques carries a certain amount of overhead in terms of time and/or resources. Our approach requires the allocation of some extra nodes for fallback scenarios, which is a resource overhead. The calculation

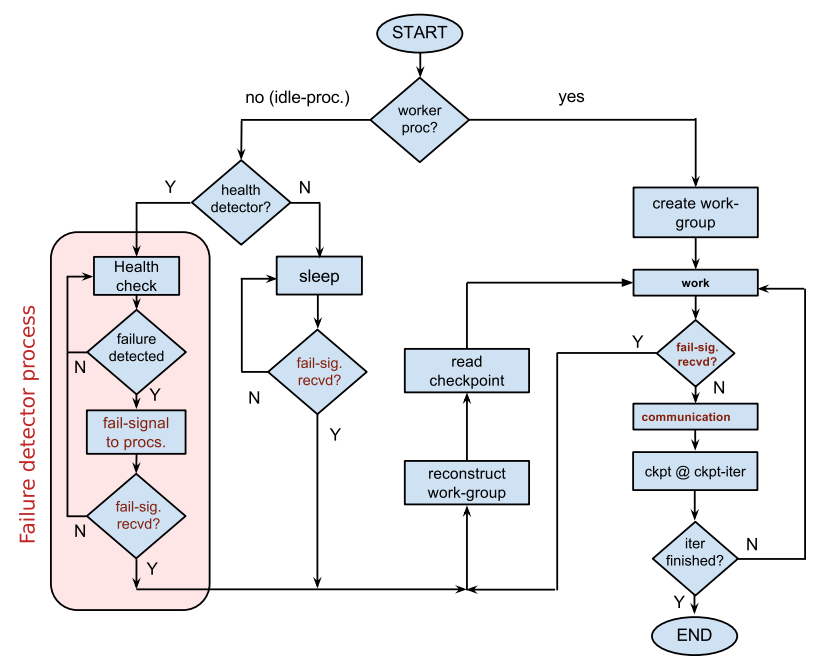

Figure 3: Algorithm flow-chart with spare processes that are kicked-in in the main program in case of failures.

of the optimal number of extra nodes for a particular case depends on several factors including job size, job duration, the MTTF of the system, etc. and is out of scope for this paper. In the following, we term overhead as the increase in application run time due to its fault tolerance capabilities.

In principle, the fault detection with global ping messages is an expensive operation. In our method, a FD process performs one-sided pings to get the global health view. On the other hand, the worker processes check for a failure acknowledgment signal from the FD process before each communication call. Thus, from the working processes' standpoint, the failure detection mechanism adds very little cost to the overall runtime of the algorithm. As we shall see in Sect. VI] this cost is negligible.

The first major overhead is introduced by checkpointing. There can be two kinds of checkpoints: a global PFS-level checkpoint, and a neighbor level checkpoint. In case of a restart, the data is initialized from a consistent checkpoint. The processes must redo the work, up to the point where the actual failure happened. This is the source of the second overhead and depends on the instance where the failure occurred between two checkpoints.

In case of a failure, all processes go through the failure detection and recovery stage of the program. This constitutes the third form of overhead and can be decomposed into three categories:

- Failure detection overhead/communication with failed processes $\left(\mathrm{OH}_{F 1}\right)$ : This is the time it takes for the FD process to successfully detect and acknowledge the failures to other processes. Meanwhile, the healthy processes trying to communicate with the failed processes end up in timeout-based returns unless a failure acknowledgment is received.

- Rebuilding of work group $\left(\mathrm{OH}_{F 2}\right)$ : This step involves the creation of a new worker group, replacing failed processes (shown in Listing. 2). Due to the blocking nature of the gaspi_group_commit() procedure, this overhead is nonnegligible. 
- Reinitialization of data $\left(\mathrm{OH}_{F 3}\right)$ : After the new work group is formed, the data structure gets initialized from the last consistent checkpoint. If the checkpoint is not available on the local node, it is fetched from the neighbor node of the failed process by the checkpoint library.

In the next sections, we perform benchmarks and get a quantitative notion of the above mentioned overheads in a practical scenario.

\section{EXPERIMENTAL FRAMEWORK}

We use the Lanczos algorithm to demonstrate the usage of our fault tolerance technique. The Lanczos algorithm is an iterative scheme to find eigenvalues of a sparse matrix. We use it to find the low-lying eigenvalues of a test matrix. Listing 1 shows the pseudo-code of the algorithm. Each iteration calculates the new Lanczos vectors, $\alpha$, and $\beta$. After obtaining the $\alpha$ and $\beta$ values of each iteration, the approximated minimum eigenvalues are determined using the QL method and are checked against a convergence criterion. The checkpointing data consists of two consecutive Lanczos vectors, $\alpha$, and $\beta$.

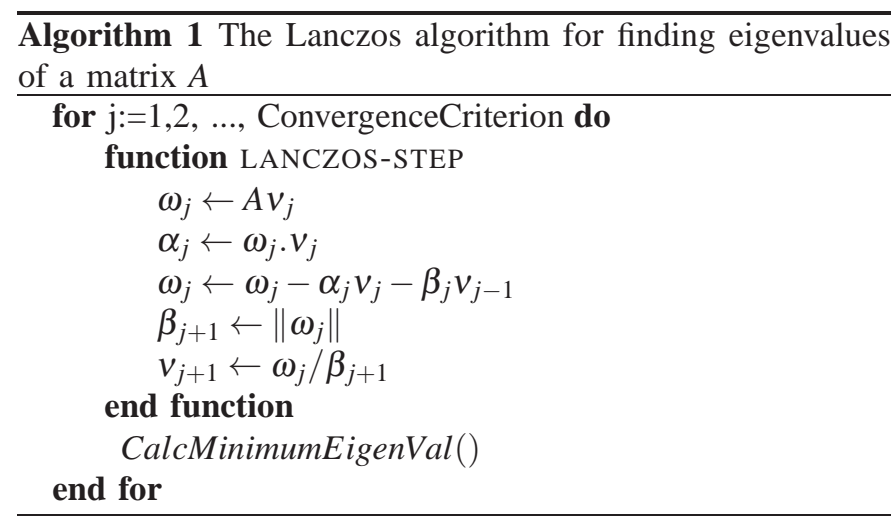

For a parallel spMVM operation, the pre-processing stage includes the setup of communication. In this stage, the spMVM operation is divided into two parts, a local part for which the process has right-hand vector values (RHS) locally available, and a remote part for which the process needs to fetch RHS values from other processes. In the pre-processing stage, each process determines the indices of the RHS that it needs from other processes. These indices are communicated to the respective processes, who then write (via one-sided GASPI communication) the RHS values of those indices before every spMVM iteration.

In the fault tolerant version of the Lanczos algorithm, each process writes a checkpoint after the pre-processing stage. This checkpoint stores information relevant for communication with other processes. After failure recovery, the rescue process reads the checkpoint of the failed process. In this way, the rescue process is informed about the communicating partners and the respective RHS indices to communicate to other processes. Every non-failing process also refreshes its list of communication partners by replacing the rank of the failed process with the new rescue process. Using this method, the program can resume the computations after failure recovery without having to perform the pre-processing step again (which would add the overall cost of recovery).
In addition to the main Lanczos application, the underlying spMVM library also needs to be prepared for fault tolerance. Each blocking communication call in spMVM library now performs a check for the failure acknowledgment signal. After the processes detect a failure signal from the FD process, no further communications are performed.

The matrix for our benchmark case arises from the quantummechanical description of electron transport properties in graphene. Graphene is a blueprint for quasi two-dimensional materials with many interesting properties and prospective applications in several fields of nanotechnology and nanoelectronics. A matrix generation library tool is used to construct the matrix on the fly. Depending upon the specified geometry size, each process allocates its own chunk of the matrix. This way, the expensive step of reading the matrix from PFS is avoided.

Testbed: All tests were done on the LiMa cluster at RRZE, whose nodes are equipped with two Intel Xeon 5650 "Westmere" chips with a base frequency of $2.66 \mathrm{GHz}$. Each node has $24 \mathrm{~GB}$ of RAM (12 GB per NUMA domain). The system has Mellanox QDR InfiniBand (IB) and GBit Ethernet interconnects.

\section{RESULTS}

In this section, we test our benchmark application with various runtime scenarios and compare their relative overhead. We have varified the recovery mechanism by killing the application processes in follwing three ways: i) Exiting the processes using 'exit(-1)' within the program ii) Using 'kill $9<$ process-id $>$ ' iii) Physically introducing a network failure. Turning off complete compute nodes was not done due to limitations of the batch queuing system which would delete the complete job owing to the dead node.

In the Lanczos algorithm, the stopping criterion depends on finding a range of eigenvalues up to a certain accuracy. For benchmarking purposes, we use a fixed number of iterations (3500) as the stopping criterion. The neighbor-node level checkpoints are taken at every 500th iteration. As discussed in the previous section, the matrix checkpoint is only stored once after its pre-processing stage at the start of the algorithm. The periodic checkpoints consist of only Lanczos vectors and the calculated eigenvalues up to the corresponding iteration. The Graphene matrix consists of $1.2 \cdot 10^{8}$ rows and columns, and $1.5 \cdot 10^{9}$ non-zeros. The global size of each checkpoint is $\approx 1.9$ GBytes. The GASPI communication timeout value is set to 1 second, after which the processes retry communication unless a failure acknowledgement is recieved from the FD process. Furthermore, the FD process performs the ping scan on all processes every 3 seconds.

Figure 4 shows the runtime of the Lanczos benchmark on 256 nodes (256 processes, 12 threads/process) in several cases. For this benchmark, the processes are killed using 'exit(-1)' at a specific iteration in order to have a deterministic redowork time. The application is started with four idle processes (nodes) reserved for failure recovery. The first case represents

\footnotetext{
${ }^{1}$ LiMa cluster at the Erlangen Regional Computing Center (RRZE): http:// www.hpc.rrze.fau.de/systeme/lima-cluster.shtml
} 


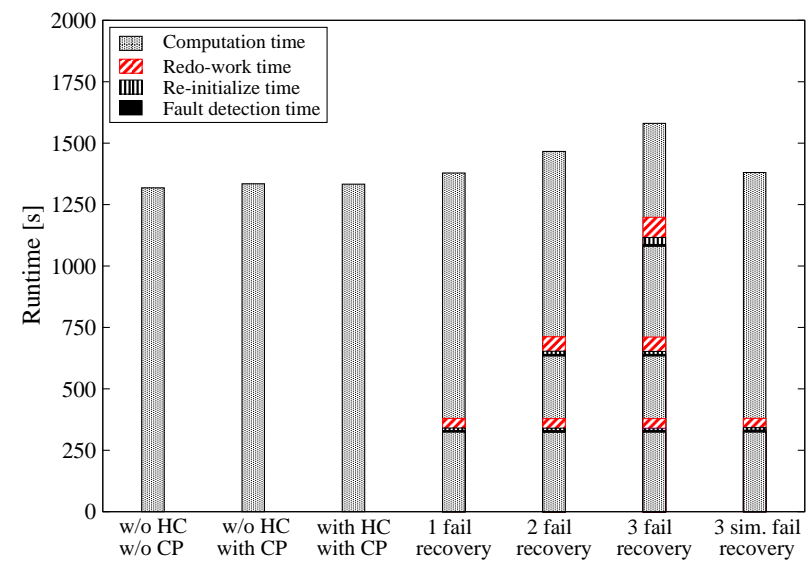

Figure 4: Various runtime scenarios of Lanczos application on 256 nodes. Each failure recovery cost $\approx 17$ seconds.

the application runtime where no health check is performed and no checkpoints are taken ('w/o HC, w/o CP' bar). This runtime marks the baseline case of our overhead study. The neighbor-node level checkpoints add negligible overhead of around $0.01 \%$ ('w/o HC, with $\mathrm{CP}$ ' bar). Furthermore the case of health check adds no additional overhead ('with HC, with $\mathrm{CP}^{\prime}$ bar). Significant overhead only appears in case of failure recovery scenarios. Each failure adds approximately 64 seconds to the total runtime of the application. The average failure detection takes $\approx 7$ seconds on 256 nodes. The 'reinitialization' overhead, which includes group reconstruction and checkpoint reading causes $\approx 10$ seconds on average. Thus a failure detection and recovery costs up to around 17 seconds. The redo-work time constitutes a major part of the total overhead. The average time for redo-work is the time between two successive checkpoints. Owing to a good checkpoint strategy with very low overhead, the checkpoint frequency can be increased which will lead to the reduction of redowork time. Recovery from more failures adds approximately proportional overhead as shown in Fig. 4 with two and three recovery case runtimes. In practice, a likely scenario is to have multiple failures simultaneously (e.g., failure of a node with multiple processes). Thus, we have used a threaded FD process (with 8 threads) to check the state of more than one process by monitoring one-sided pings in parallel on different communication queues. This is highlighted in ' 3 sim. fail recovery' case, where three simultaneous failures are detected with the overhead of one failure detection.

It is important to observe and analyse the scaling behavior of a fault detection mechanism. Table \ shows the ping scan time of the FD process without any failure. The FD process takes approximately $1 \mathrm{~ms}$ to perform a ping with each healthy process. The table also shows the failure detection and acknowledgement time in case of a failure. For this benchmark, one randomly selected process is terminated using 'kill -9 $<$ process-id $>$ ' at a random instance during the application run. The time is measured between the instance of fault injection and the acknowledgement of failures by the processes. The experiments show a good scalability of fault detection and acknowledgement method upto 256 nodes. It is important to

\begin{tabular}{|c|c|c|c|c|c|c|}
\hline Num. of Nodes & 8 & 16 & 32 & 64 & 128 & 256 \\
\hline \hline $\begin{array}{c}\text { Avg. ping scan } \\
\text { time[s] }\end{array}$ & 0.010 & 0.018 & 0.036 & 0.067 & 0.129 & 0.255 \\
\hline $\begin{array}{c}\text { Failure } \\
\begin{array}{c}\text { detection and } \\
\text { ack. time[s] }\end{array}\end{array}$ & $\begin{array}{c}4.9 \\
\pm 0.7\end{array}$ & $\begin{array}{c}5.3 \\
\pm 0.7\end{array}$ & $\begin{array}{c}5.5 \\
\pm 0.8\end{array}$ & $\begin{array}{c}4.3 \\
\pm 0.7\end{array}$ & $\begin{array}{c}5.7 \\
\pm 1.2\end{array}$ & $\begin{array}{c}5.3 \\
\pm 0.8\end{array}$ \\
\hline
\end{tabular}

Table I: The average ping scan time of the FD process and the failure detection time (and standard deviation using 10 runs) with respect to the number of nodes.

mention that this time is the summation of the fault detecion overhead $\mathrm{OH}_{F 1}$ (as explained in previous section) as well as the delay of 3 seconds between two ping scans.

\section{RELATED WORK}

The prototype implementation of ULFM using OpenMPI has produced similar work to ours in terms of failure recovery strategies and/or performance evaluation.

Bland et al. have implemented a fault tolerant Monte Carlo Communication Kernel proxy-app using ULFM in [16]. They have demonstrated two recovery modes of the application, a classic checkpoint/restart approach and an alternative solution in which the critical data is stored on neighboring nodes after each iteration. The recovery is done by spawning an additional process which reads the data from the failed process's neighboring node. In [17], a different approach of data recovery is implemented to develop a fault tolerant Multi-Level Monte Carlo based application using ULFM. The data from failed processes are altogether discarded and only survivors' data is used to complete the application further. Depending on the number of failures, the estimation error deteriorates the quality of results. A similar strategy was pursued by Ali et al. to develop a 2D PDE solver in [18]. They compare three alternative data recovery approaches based on $\mathrm{C} / \mathrm{R}$ and approximation techniques. All mentioned studies have found ULFM to have acceptable overhead for failure recovery. In [15], Laguna et al. have conducted a broad and critical evaluation of ULFM. They have first discussed preferable recovery modes based on the nature of the applications and then performed a case study on a ULFM-based implementation of a fault-tolerant molecular dynamics application (ddcMD). They have found the time of revoking and shrinking the communicator to be increasing linearly with increasing number of nodes. In the end, they have suggested improvements to ULFM to make it an attractive option for application developers.

The ULFM studies are based on the detection of faults by communication failure between processes. No explicit failure detection methods are used. In [19], K. Kharbas et al. have evaluated two kinds of fault detector mechanisms for MPI applications based on periodic and sporadic probing. The probing is based on ping-pong style messaging. They have only reported the overhead for failure-free cases which ranges from $1 \%$ to $21 \%$, averaging around $10 \%$ for NAS-parallel benchmarks. The fault detector overhead is negligible when separate background processes are used to check failures on the same set of nodes and the main application. In this case the background processes use Gigabit Ethernet instead of the 
Infiniband network used by the application, but this is not a suitable design as a large category of faults are network related. They conclude that a separate periodic fault detection mechanism is a superior method as compared to an sporadic approach.

\section{SUMMARY}

We have implemented a fault-tolerant version of a parallel Lanczos algorithm using the GPI-2 communication library with custom extensions. An explicit failure detector (FD) was designed to monitor the health of all processes. The FD process performs periodic one-sided pings to monitor the health state of all processes. We have used the idea of spare processes in combination with a checkpoint/restart mechanism for the replacement of failed processes. One of these spare processes is designated to be the FD process, whereas the rest stays idle until a failure occurs and they join the worker processes. The efficiency of checkpoints is optimized by using a neighbor node-level checkpoint/restart library which is also fault tolerance aware.

The benchmark performed on 256 nodes show that having an explicit FD causes no overhead to the working processes. The recovery from a single failure causes a total overhead of around 64 seconds, a large part which comes from the redo-work time. The failure detection itself takes $\approx 7 \mathrm{sec}-$ onds, whereas the communication reconstruction and data re-initialization from the checkpoint add $\approx 10$ seconds of overhead. Each additional failure adds similar cost. On the other hand, if multiple failures happen simultaneously (likely scenario for node failures), they have the potential to be detected at the cost of a single failure. Our failure detection mechanism shows good scaling behaviour on the scaling test performed up to 256 nodes.

Discussion and Future work: The fault tolerance research is in its infancy stages regarding application-driven fault tolerance. Only having FT communication is not nearly enough. Many other components are needed to build an FT application in its true sense, e.g., failure information propagation to healthy processes, communication reconstruction after failure, data recovery method, etc. These add extra burden in terms of application development.

More fault tolerance related functionalies can be included within or on top of the GASPI framework in order to simplify the development of fault tolerant codes. These include failed processes acknowledgment by groups, the replacement of failed processes by a newly spawned ones, etc. Furthermore, functionality can be provided to assign the identity of the failed process to the newly spawned/replaced process.

Our future work is targeted to remove the current limitations of our approach and making the technique more general and user friendly. The redundancy approach can be implemented to make the FD process fault tolerant. We also plan to compare this fault tolerance approach with the Open MPI's ULFM functionality.

\section{ACKNOWLEDGMENT}

This work was partly supported by the German Research Foundation(DFG) through the Priority Programme 1648
"Software for Exascale Computing"(SPPEXA) and partly by Federal Ministry of Education and Research (BMBF) under project "A Fault Tolerant Environment for Peta-scale MPIsolvers" (FETOL) (grant No. 01IH11011C).

\section{REFERENCES}

[1] TOP500, “TOP500 supercomputing sites," http://top500.org.

[2] M. Snir et al., "Addressing failures in exascale computing," International Journal of High Performance Computing Applications, vol. 28, no. 2, p. 127 171, May 2014.

[3] J. Dongarra, "Emerging Heterogeneous Technologies for High Performance Computing," http://www.netlib.org/utk/people/JackDongarra/ SLIDES/hcw-0513.pdf, May 2013.

[4] J. Daly et al., "Inter-Agency Workshop on HPC Resilience at Extreme Scale," http://institute.lanl.gov/resilience/docs/ Inter-AgencyResilienceReport.pdf, Tech. Rep., Feb. 2012.

[5] B. Schroeder and G. A. Gibson, "Understanding failures in petascale computers," Journal of Physics: Conference Series, 2007.

[6] N. El-Sayed and B. Schroeder, "Reading between the lines of failure logs: Understanding how HPC systems fail," in Proceedings of the 201343 rd Annual IEEE/IFIP International Conference on Dependable Systems and Networks (DSN), ser. DSN '13. Washington, DC, USA: IEEE Computer Society, 2013, pp. 1-12. [Online]. Available: http://dx. doi.org/10.1109/DSN.2013.6575356

[7] J. Hursey, "Coordinated Checkpoint/Restart Process Fault Tolerance for MPI Applications on HPC Systems," Ph.D. dissertation, Indiana University, Bloomington, IN, USA, July 2010.

[8] W. Bland, G. Bosilca, A. Bouteiller, T. Herault, and J. Dongarra, "A proposal for User-Level Failure Mitigation in the MPI-3 Standard," http://www.mcs.anl.gov/ wbland/pdf/Bland_2012_A_proposal_ for_User-Level_Failure_Mitigation_in_the_MPI-3_standard.pdf, 2012.

[9] W. Bland, A. Bouteiller, T. Herault, G. Bosilca, and J. Dongarra, "Post-failure recovery of MPI communication capability: Design and rationale," International Journal of High Performance Computing Applications, vol. 27, no. 3, pp. 244-254, 2013. [Online]. Available: http://hpc.sagepub.com/content/27/3/244.abstract

[10] GPI2 website, http://www.gpi-site.com/gpi2/.

[11] GASPI project website, http://www.gaspi.de/en/project.html

[12] A. Moody et al., "Design, Modeling, and Evaluation of a Scalable Multilevel Checkpointing System," in Proceedings of the 2010 ACM/IEEE International Conference for HPC, Networking, Storage and Analysis, Washington,DC, USA, 2010, pp. 1-11.

[13] K. Sato et al., "Design and modeling of a non-blocking checkpointing system," in Proceedings of the International Conference on High Performance Computing, Networking, Storage and Analysis. Los Alamitos, CA, USA: IEEE Computer Society Press, 2012, pp. 19:1-19:10.

[14] T. D. Chandra and S. Toueg, "Unreliable failure detectors for reliable distributed systems," J. ACM, vol. 43, no. 2, pp. 225-267, Mar 1996.

[15] I. Laguna, D. F. Richards, T. Gamblin, M. Schulz, and B. R. de Supinski, "Evaluating user-level fault tolerance for mpi applications," in Proceedings of the 21st European MPI Users' Group Meeting, ser. EuroMPI/ASIA '14. New York, NY, USA: ACM, 2014, pp. 57:57-57:62. [Online]. Available: http://doi.acm.org/10.1145/2642769. 2642775

[16] W. Bland, K. Raffenetti, and P. Balaji, "Simplifying the recovery model of user-level failure mitigation," in Proceedings of the 2014 Workshop on Exascale MPI, ser. ExaMPI '14. Piscataway, NJ, USA: IEEE Press, 2014, pp. 20-25.

[17] S. Pauli, M. Kohler, and P. Arbenz, "A fault tolerant implementation of Multi-Level Monte Carlo methods," in Parallel Computing: Accelerating Computational Science and Engineering (CSE), Proceedings of the International Conference on Parallel Computing, ParCo 2013, 2013, pp. 471-480. [Online]. Available: http://dx.doi.org/10.3233/ 978-1-61499-381-0-471

[18] M. M. Ali, J. Southern, P. Strazdins, and B. Harding, "Application Level Fault Recovery: Using Fault-Tolerant Open MPI in a PDE Solver,' in Parallel Distributed Processing Symposium Workshops (IPDPSW), 2014 IEEE International, May 2014, pp. 1169-1178.

[19] K. Kharbas, D. Kim, T. Hoefler, and F. Mueller, "Assessing HPC failure detectors for MPI jobs," in Proceedings of the 20th Euromicro International Conference on Parallel, Distributed and Network-Based Processing, PDP 2012, Munich, Germany, February 15-17, 2012, 2012, pp. 81-88. [Online]. Available: http://dx.doi.org/10.1109/PDP.2012.11 